\title{
Inductive electromagnetic effects in solar current-carrying magnetic loops
}

\author{
M. Khodachenko ${ }^{1}$, G. Haerendel ${ }^{2}$, and H. O. Rucker ${ }^{1}$ \\ 1 Space Research Institute, Austrian Academy of Sciences, Schmiedlstraße 6, 8042 Graz, Austria \\ 2 International University Bremen Campus Ring 1, 28759 Bremen, Germany \\ e-mail: hae@iu-bremen.de
}

Received 20 December 2000 / Accepted 28 January 2003

\begin{abstract}
Effects of electromagnetic inductive interactions in groups of slowly growing current-carrying loops are studied. Each loop is considered as an equivalent electric circuit with variable resistivity and inductive coefficients. These parameters depend on the geometry of the loop, its position with respect to neighboring loops, as well as on the plasma temperature and density in the magnetic tube. By means of such a model the process of generation of currents and temperature change in coronal loops moving relative to each other, and their dynamic interaction were studied. There are three main results of this analysis. First, the possibility of a relatively quick development of a significant longitudinal current in a rising and initially current-free magnetic loop is demonstrated. Second, the processes of fast, flare-like, plasma temperature increase in inductively connected growing loops with high enough currents, $\sim 10^{10}-10^{11} \mathrm{~A}$, as well as run-away electrons acceleration in the loops by inductive electric fields are modelled. And third, based on the analysis of a ponderomotoric interaction of current-carrying magnetic loops, conditions for their oscillations or a fast change of the loops inclination, possibly resulting in their coalescence and magnetic reconnection, are studied. The main characteristics of the oscillatory dynamics of a loop were calculated.
\end{abstract}

Key words. Sun: magnetic fields - Sun: flares - Sun: oscillations - Sun: corona

\section{Introduction}

Observations of a vector magnetic field on the Sun provide sufficient information to determine a vertical component of $\operatorname{rot} \boldsymbol{B}$ and hence to identify the vertical component of a current flowing from below the photosphere into the corona (Moreton \& Severny 1968; Ding et al. 1987; Hagyard 1989; Canfield et al. 1993; Wang H. et al. 1994; Gary \& Demoulin 1995; Wang J. et al. 1996; Leka et al. 1996; Hardy et al. 1998). The observed currents in active regions can reach values of up to $10^{11}-10^{12} \mathrm{~A}$. Usually, the distribution of the current, which is deduced from vector magnetograms, can be presented as a set of current-carrying loops centered on a neutral line (Hagyard 1989; Canfield et al. 1990, 1993; Melrose 1991). The data indicate that the current in these loops is unneutralized, i.e. it flows from one footpoint of the magnetic loop to the other with no evidence for a return current flowing through the corona. The return current should naturally appear when the current along the loop is generated by a subphotospheric twisting motion (Spicer 1982; Jones \& Galloway 1993; Melrose 1991). In spite of the data on the vector magnetic field, which suggest that the currents in coronal magnetic loops are unneutralized, there appears to be no theory explaining how such unneutralized currents could be set up after a magnetic loop emerges

Send offprint requests to: M. Khodachenko, e-mail: Maxim.Khodachenko@oeaw.ac.at from below the photosphere. Melrose $(1991,1995,1997)$ supposes that the currents in coronal loops must already be flowing when a flux tube emerges. It is assumed there that the dynamo region is located deep below the photosphere, near the base of the convection zone (see also discussion in Melrose 1996; Parker 1996). In the present paper we consider a mechanism which could influence the currents flowing in the coronal magnetic loops without acting upon the subphotospheric dynamo. It is based on the effects of the inductive electromagnetic interaction of relatively moving (rising and growing) neighboring magnetic loops. To study the inductive interaction of the loops we employ a circuit model of a current-carrying magnetic loop (Spicer 1982; Melrose 1995; Zaitsev \& Khodachenko 1997; Khodachenko \& Zaitsev 1998).

Observations of solar flares in soft X-ray, hard X-ray, and radio wavelengths indicate that quite often multiple loops are involved in a flare process (Hanaoka 1996, 1997; Nishio et al. 1997; Aschwanden et al. 1999a). It follows from a combination of microwave, soft X-ray and magnetogram data that a large number of solar flares occurs in regions where a new magnetic loop emerges and interacts with the existing loops. This phenomenon is known as so-called "interacting flare loops". This term is traditionally used when due to the unequal vertical motion of magnetic loops, they come into close contact and reconnect with each other. During the process of reconnection the original magnetic structure evolves into a new state with 
a lower magnetic energy, whereas the rest of the magnetic energy is released by the flare (Melrose 1997; Hardy et al. 1998; Aschwanden et al. 1999a).

Regarding this picture of a flare, we note that none of the current-carrying flux tubes can be magnetically isolated from its surroundings. In any realistic geometry in which the current is confined to a current channel, it generates a magnetic field outside the channel. This implies that current-carrying magnetic loops should interact with each other through their magnetic fields. The simplest way to take into account this interaction consists in the application of the equivalent electric circuit model of a loop which includes a time-dependent inductance, mutual inductance, and resistance. Relative motion of inductively coupled current-carrying magnetic loops causes the appearance of strong inductive electromotive forces in their circuits. These electromotive forces influence the currents in the loops from the very beginning of their motion and well before the loops contact each other and reconnect. They could even appear as a current source in a loop.

Besides, each pair of current-carrying magnetic loops interacts through the magnetic field of one and the current of another by a $\frac{1}{c}[\boldsymbol{j} \times \boldsymbol{B}]$ force, which couples magnetic loops dynamically. The new TRACE observations give a nice view of the coronal loop dynamics: growth motions, oscillations, meandering and twisting (Handy et al. 1999). The oscillations of the loops are usually modelled as standing or propagating MHD wave modes (Aschwanden et al. 1999b; De Moortel et al. 2000). At the same time the explanation of the observed decay of the loop oscillations within the frame of such a model requires very high unrealistic wave energy dissipation rates (Nakariakov et al. 1999). In this work we pay attention to the dynamic coupling of current-carrying loops and interpret their oscillations in terms of the ponderomotoric interaction of currents.

The electromagnetic inductive coupling of the solar atmosphere also plays a significant role in stabilizing possible kink instabilities of current channels in coronal magnetic loops. this increases the ability of the whole current system of an active region to store magnetic energy.

The equivalent electric circuit models, considered in this paper, are strong idealizations of the real coronal magnetic loops. Thus, first of all we say a few words about the possibility of their application and limitations. The circuit model usually involves highly simplified geometry assumptions and is obtained by integrating an appropriate form of Ohm's law for a plasma over a circuit (see Spicer 1982; Melrose 1995). The actual spatial distribution of the current in the loop is replaced with the current $I=c r_{0} B_{0}$ homogeneously distributed over the cross-section of the current channel with the radius $r_{0}$. A simple circuit model ignores the fact that changes of the magnetic field propagate in plasma at the Alfvén speed $V_{\mathrm{A}}$ (Melrose 1992; Zaitsev et al. 1978). This means that the circuit equations correctly describe temporal evolution of the currents in a solar coronal magnetic current-carrying structure only on a timescale longer than the Alfvén propagation time. More generally, the electric circuit model tends to emphasize the global circuit, obscuring the effects of the ambient plasma and details of the magnetic structures. At the same time, the main advantage of this approach is that it allows us to simplify the complex magnetic field and current systems in the solar active region while still incorporating the appropriate physics.

In order to create more realistic models, in some cases below we take into account the fact that due to the temporal change of the lengths of the inductively coupled current-carrying loops and associated change of inductive and resistive parameters of their equivalent electric circuits, a temporal change of the currents in the circuits takes place, which in its turn disturbs the initial thermal equilibrium of the loops. As a result, the temperature of the plasma in the interacting loops starts to change. This finally affects the value of the plasma conductivity and the equivalent resistances in the circuits. Thus, the evolution of each current-carrying loop should be described by two equations: 1) the equation for an equivalent electric circuit and 2) an appropriate form of the energy equation (Khodachenko \& Zaitsev 1998).

\section{Inductive currents in coronal magnetic loops}

\subsection{General features of the coronal circuit, and dynamics of current}

The equation for the equivalent electric circuit, corresponding to a coronal part of a separate (but not isolated from surroundings) magnetic loop can be written in the following form:

$I R=U_{0}-\frac{1}{c^{2}} \frac{\mathrm{d}}{\mathrm{d} t}(L I)-\frac{1}{c} \frac{\mathrm{d} \Psi_{\mathrm{ext}}}{\mathrm{d} t}$

Here $I$ is the total current flowing along the loop, and $U_{0}$ is the potential drop between the loop's footpoints. The quantity $U_{0}$ can sometimes be associated with an external electromotive force applied to the circuit. The parameters $L$ and $R$ are inductance and resistance of the coronal part of the loop respectively. The external magnetic flux through the circuit of the loop, $\Psi_{\text {ext }}=B_{\perp} S_{\text {loop }}$, is determined by the normal component of the external magnetic field, $B_{\perp}$ with regard to the plane of the loop, as well as by the loop geometry. Here $S_{\text {loop }}$ is the area covered by the loop. The term including an external magnetic flux can be considered as an additional electromotive force applied to the circuit. In the case of inductively coupled electric circuits this term appears in the circuit equations as a mutual inductance electromotive force $\mathcal{E}_{i}^{\text {ind }}=\frac{1}{c^{2}} \frac{\mathrm{d}}{\mathrm{d} t}\left(M_{i j} I_{j}\right), i \neq j$. Here $I_{j}$ is an electric current in the neighboring $j$-th circuit, which interacts with a current in a given $i$ th circuit. $M_{i j}=M_{j i}$ are the mutual inductance coefficients.

An important feature of the equivalent electric circuit, for a coronal magnetic loop, consists of the large value of inductance of the loop. Approximating the magnetic loop by a thin torus with the main radius $R_{\text {loop }}$ and a small radius $r_{0}$, so that $R_{\text {loop }} \gg$ $r_{0}$ (Alfvén \& Carlqvist 1967; Melrose 1997) we can calculate its inductance as (Landau \& Lifshitz 1960a, p. 139)

$L=4 \pi R_{\text {loop }}\left(\ln \frac{8 R_{\text {loop }}}{r_{0}}-\frac{7}{4}\right)$.

For $R_{\text {loop }}=5 \times 10^{9} \mathrm{~cm}$ and $r_{0}=10^{8} \mathrm{~cm} \mathrm{Eq.} \mathrm{(2)} \mathrm{gives}$ $L=2.66 \times 10^{11} \mathrm{~cm} \approx 100 \mathrm{H}$. An electric circuit with a large inductance has a very big characteristic time $\tau=\frac{L}{R c^{2}}$, which 
represents the time scale of the current variation in the circuit after the switch on/off of an external electromotive force or resistance. Estimating the resistance of a coronal loop as $R=\frac{2 R_{\text {loop }}}{r_{0}^{2} \sigma(T)}$, where $\sigma(T)=9 \times 10^{6} T^{3 / 2}$ is plasma conductivity, one obtains for magnetic loops, containing a plasma with the temperature $10^{6} \mathrm{~K}$, a characteristic time $\tau$ that is of the order of $1.6 \times 10^{4}$ years. Thus, no variation of the current can be expected on the time scales like the duration of a flare, or the life time of an active region. At a first glance, this fact puts unresolvable barriers in the way of dynamic circuit models of solar magnetic loops and associated flares. However, taking into account the process of a magnetic loop rising, i.e., growing in height and length, which is directly connected (see Eq. (2)) with the change of inductance of the loop, leads to the appearance of one more characteristic time scale $\tau_{L}=\frac{L}{\mathrm{~d} L / \mathrm{d} t}$. It is this time scale which finally determines the duration of the current change in the circuit.

To demonstrate this let us consider the dynamics of a current in a circuit described by the following equation

$I R_{0}=U_{0}-\frac{1}{c^{2}} \frac{\mathrm{d}}{\mathrm{d} t}(L I)$.

Let us assume for definiteness that the inductance of the circuit changes linearly, i.e., $L(t)=L_{0}+a \cdot t$, whereas the resistance, $R_{0}$, and $U_{0}$ remain constant. As the initial condition we take $I(t=0)=I_{0}=\frac{U_{0}}{R_{0}}$. In this case Eq. (3) has the solution

$I(t)=\frac{I_{0}}{1+\delta}\left(\frac{L_{0}^{1+\delta}}{\left(L_{0}+a t\right)^{1+\delta}}+\delta\right)$,

where $\delta=\frac{R_{0} c^{2}}{a}$. One can see from Eq. (4) that the dynamics of the current is completely defined by the dynamics of inductance. From the requirement that the inductance changes sufficiently quickly, in comparison with the characteristic time scale of the circuit, $\tau_{L} \ll \tau$, we have $\delta \ll 1$. Thus, Eq. (4) can be reduced to

$I(t) \approx \frac{U_{0}}{R_{0}}\left(\frac{L_{0}}{L_{0}+a t}+\delta\right)$.

The above example illustrates the connection between a temporal change of the inductance in the electric circuit and the dynamics of the current. However, this elementary case can not be directly applied for the description of the evolution of a longitudinal electric current in a rising solar magnetic loop.

\subsection{Dynamics of current in a rising magnetic loop}

The main difference between an equivalent electric circuit model of a rising coronal magnetic loop and the example considered in Sect. 2.1, consists not only of a temporal dependence of the resistance of the circuit of the growing magnetic loop $R=\frac{l_{\text {loop }}(t)}{S \sigma}$ (where $l_{\text {loop }}(t)$ is the length of the loop, changing in time and $S$ is the cross-section of the current channel), but also in a nonstationarity (in the general case) of the inductive electromotive force, $-\frac{1}{c} \frac{\mathrm{d} \Psi_{\text {ext }}}{\mathrm{d} t}$, caused by a change of magnetic flux $\Psi_{\text {ext }}(t)=B_{\perp} S_{\text {loop }}(t)$ through the circuit.

As a next step, approaching the real situation of a growing coronal magnetic loop, which is connected inductively to the complex magnetic environment of an active region, we consider a loop rising in a constant homogeneous background magnetic field. The normal with respect to the plane of the loop component of the magnetic field we denote here as $B_{\perp}=B_{0}$. Let us assume that initially there was no current in the loop, i.e. $I(t=0)=0$. This means that in Eq. (1) one should take $U_{0}=0$ during the evolution of the loop. This would be equivalent to an infinite capacitance $C_{\infty}$ switched between the footpoints of the loop, so that $\frac{\mathrm{d} U_{0}}{\mathrm{~d} t}=\frac{I}{C_{\infty}} \approx 0$.

The main purpose of our analysis in this subsection is to show a simple example of how the inductive electromotive force $\mathcal{E}^{\text {ind }}$, caused by the temporal change of an external magnetic flux $\Psi_{\text {ext }}=B_{\perp} S_{\text {loop }}$, through the circuit of a slowly evolving magnetic loop can result in the appearance of a significant longitudinal current in the loop. By this, since

$\mathcal{E}^{\text {ind }}=-\frac{1}{c} \frac{\mathrm{d} \Psi_{\mathrm{ext}}}{\mathrm{d} t}$

it is not very important which particular process is responsible for the temporal change of the flux $\Psi_{\text {ext }}$. In principle, the magnetic loop may not move at all and only a new magnetic flux providing a non-zero $B_{\perp}(t)$ emerges below it. Equally, one can consider a situation when the magnetic loop grows up and the space below it is filled with a magnetic field newly emerging from below the photosphere. In all these cases we shall have similar temporal behaviour of the current in the loop.

Again using the thin torus approximation for the coronal loop, Eq. (1) can be written in the following form

$\frac{\mathrm{d}}{\mathrm{d} t}\left(I R_{\text {loop }}\left(\operatorname{Ln} \frac{8 R_{\text {loop }}}{r_{0}}-\frac{7}{4}\right)\right)=-a_{1} I R_{\text {loop }}-a_{2} R_{\text {loop }} \frac{\mathrm{d} R_{\text {loop }}}{\mathrm{d} t}$,

where

$a_{1}=\frac{c^{2}}{2 \pi \sigma(T) r_{0}^{2}}, \quad a_{2}=\frac{B_{0} c}{2 \pi}$.

Here $R_{\text {loop }}(t)$ and $r_{0}$ are the main radius of the loop and the radius of its cross-section respectively. Note that the term with the logarithm on the left hand side of Eq. (6) changes slowly when $R_{\text {loop }}(t)$ varies in time. This term can be taken out as a constant factor $\approx\left(\operatorname{Ln} \frac{8 R_{\text {loop }}^{0}}{r_{0}}-\frac{7}{4}\right)$ from the time derivative. In this case we obtain an approximate equation

$\frac{\mathrm{d}}{\mathrm{d} t}\left(I R_{\text {loop }}\right)=-b_{1} I R_{\text {loop }}-b_{2} R_{\text {loop }} \frac{\mathrm{d} R_{\text {loop }}}{\mathrm{d} t}$,

where

$b_{1}=\frac{c^{2}}{2 \pi 9 \times 10^{6} T^{3 / 2} r_{0}^{2}}\left(\operatorname{Ln} \frac{8 R_{\text {loop }}^{0}}{r_{0}}-\frac{7}{4}\right)^{-1}$,

$b_{2}=\frac{B_{0} c}{2 \pi}\left(\operatorname{Ln} \frac{8 R_{\text {loop }}^{0}}{r_{0}}-\frac{7}{4}\right)^{-1}$.

Assuming the linear law for the temporal change of the radius of the loop $R_{\text {loop }}(t)=R_{\text {loop }}^{0}+a t$, Eq. (7) can be solved analytically. For the initial condition $I(t=0)=0$ the solution of Eq. (7) is

$$
\begin{aligned}
I(t)= & -\frac{a b_{2}}{b_{1}^{2}\left(R_{\text {loop }}^{0}+a t\right)} \mathrm{e}^{-b_{1} t} \\
& \times\left(b_{1} R_{\text {loop }}^{0}\left[\mathrm{e}^{b_{1} t}-1\right]+a\left[1+b_{1} t \mathrm{e}^{b_{1} t}-\mathrm{e}^{b_{1} t}\right]\right) .
\end{aligned}
$$




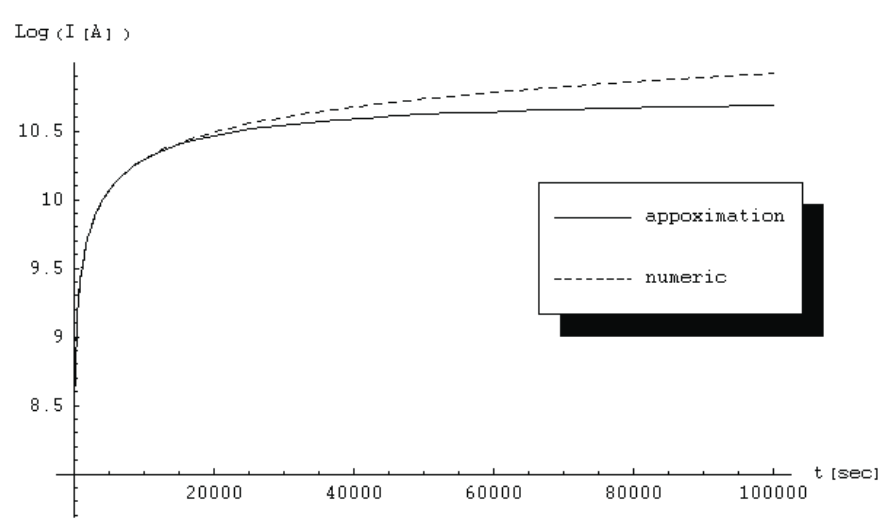

Fig. 1. Build-up of the current in the initially current-free magnetic loop, rising in a fixed background magnetic field.

Since $b_{1} t=t / \tau_{0}$, where $\tau_{0}=\frac{L_{0}}{c^{2} R_{0}}$ is the characteristic time scale of the circuit in the initial moment $(t=0)$, then $b_{1} t \ll 1$ for all reasonable times $t$. Making a series expansion of Eq. (8) in orders of $b_{1} t$ we finally obtain an approximate formula which describes the increase of the current in the rising initially current-free coronal magnetic loop

$I(t) \approx-\frac{a R_{\mathrm{loop}}^{0} b_{2} t}{R_{\mathrm{loop}}^{0}+a t}$.

This approximate formula well describes the typical features of the current evolution in the loop, such as a relatively fast build up of the current at the beginning of the loop rising and its following slower change. The sign of the current depends upon the sign of the projection of the background magnetic field onto the normal direction to the plane of the loop ( $B_{0}$ in our case). In Fig. 1 we present the dynamics of the current in a loop with $r_{0}=$ $5 \times 10^{7} \mathrm{~cm}$, and $R_{\text {loop }}^{0}=2 \times 10^{8} \mathrm{~cm}$, containing coronal plasma with $T=10^{6} \mathrm{~K}$. The loop grows with the speed $a=10^{4} \mathrm{~cm} \mathrm{~s}^{-1}$ in the background magnetic field $B_{0}=100 \mathrm{G}$, orthogonal to the plane of the loop and directed oppositely to the normal of the circuit. The dashed line in Fig. 1 corresponds to the numerical solution of the general Eq. (6), whereas the solid line is the approximate solution (9). Both solutions are very close to each other up to $t \sim 20000 \mathrm{~s}$, and only after that become sightly different. The approximate solution (9) tends asymptotically to a constant value of $I_{\mathrm{c}}=b_{2} R_{\text {loop }}^{0}=5.8 \times 10^{10} \mathrm{~A}$, whereas the numerical solution shows a slow increase of $I$.

The numerical solutions of Eq. (6) for the same parameters of the loop as above, but for different values of the rising speed $a=2.5 \times 10^{5} ; 10^{5} ; 10^{4} \mathrm{~cm} \mathrm{~s}^{-1}$ are presented in Fig. 2. The typical values obtained for the current in the loops are $10^{10}-10^{11} \mathrm{~A}$. The loops with higher rise speeds quickly get high longitudinal currents, whereas it takes a longer time for slow loops to reach the same values of the current (see Figs. 1 and 2).

\section{Interaction of two current-carrying magnetic loops}

The energetic processes in groups of inductively connected current-carrying loops were considered and modelled by

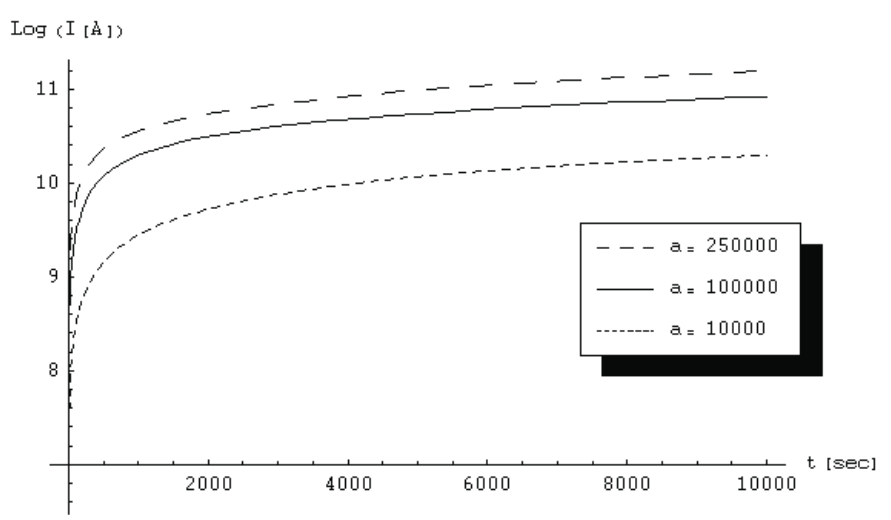

Fig. 2. Dependence of the current generated in the growing magnetic loop on the rising speed of the loop.

Khodachenko \& Zaitsev (1998). Here we consider the simpler case of the interaction of only two loops. The similarity of the situation in this elementary case with the one considered in the so-called quadrupolar magnetic reconnection model of a solar flare suggested by Melrose (1997), gives rise to a special interest in its analysis. The quadrupolar magnetic reconnection model was successfully applied for observational data by Hardy et al. (1998) and Aschwanden et al. (1999a). According to this model, the relative motion of two neighboring loops leads to their intersection and exchange of currents and flux through the process of magnetic reconnection. The main assumption of the quadrupolar magnetic reconnection model of a flare is that the strengths of currents flowing through the footpoints of the loops can not change significantly over the time scale of a flare. This follows from the fact that the duration of a flare is much shorter than the time required to affect the source of the current, supposed to be located deep under the photosphere (the Alfvén propagation time, Melrose 1992, 1995, 1997). This constraint implies that the energy release in the flare is due to a redistribution of the current and flux above the photosphere, while the net current flowing into and out of the corona at the footpoints remains fixed. The quadrupolar magnetic reconnection model relates the geometry of the footpoints of the loops and the currents within the loops to the total energy released by the flare. By this, the dominant contribution to the energy release is due to the change of magnetic energy associated with the redistribution of the currents between the foot points of the inductively coupled loops. In particular, the magnetic energy stored in the system before and after the reconnection of the loops is calculated, and the energy difference is considered as a possible flaring energy release.

Regarding this model of a flare we note that the relative motion of the inductively coupled current-carrying loops influences the dynamics of the currents in these loops from the very beginning of their motion. The relative motion of the loops creates significant inductive electromotive forces in their electric circuits. These inductive electromotive forces themselves could appear as a powerful source for changing the currents without influencing deep subphotospheric sources. Change of the currents in the loops disturbs their initial thermal equilibrium, and results in a change of the plasma temperature, which in its turn influences the resistivity of the circuit and the radiative energy 
losses. Thus, relative motion of inductively connected currentcarrying magnetic loops causes complex dynamics of not only currents, but also plasma parameters in the loops.

The inductive interaction between current-carrying loops implies that changes of longitudinal current and reorganization of the magnetic field are inter-related processes which involve the plasma inside and outside of the magnetic tube. The variation of the magnetic field outside of the magnetic tube, connected with the change of the state of the tube itself, happens after a characteristic time $\tau_{\mathrm{A}}=r / V_{\mathrm{A}}$, where $r$ is the distance from the given point to the evolving magnetic tube and $V_{\mathrm{A}}$ is the Alfvén speed (Zaitsev et al. 1978; Melrose 1992). Moreover, the characteristic time for the internal dynamics of the plasma (relaxation to an equilibrium steady state) in the magnetic tube is of the order of several times $\tau_{\mathrm{A} 0}=r_{0} / V_{\mathrm{A}}$, where $r_{0}$ is the transverse scale of the tube. Taking these circumstances into account we consider sufficiently slow loop motion so that at each given moment of time we can assume a quasi-steady state for the plasma and fields inside and around the loops. Thus, below, in our models we neglect the internal dynamics of plasma in magnetic tubes, which was studied by Khodachenko (1996), and consider the density $n$ to be a constant.

In the model of the interaction of two inductively coupled, moving, current-carrying loops, which we consider in this section, each of the loops is described by two equations (Khodachenko \& Zaitsev 1998), the equation for the electric circuit of the loop

$I_{i} R_{i}=U_{0 i}-\frac{1}{c^{2}}\left(L_{i} \dot{I}_{i}+I_{i} \dot{L}_{i}+M_{i j} \dot{I}_{j}+I_{j} \dot{M}_{i j}\right)$

and the energy equation

$\dot{T}_{i}=\frac{1-\gamma}{2 n_{i} k_{B}}\left(n_{i}^{2} Q\left(T_{i}\right)-\frac{j_{i}^{2}}{\sigma\left(T_{i}\right)}-H_{i}\right)$

where indexes $i, j$ indicate the loop number ( 1 or 2 ). The dot in Eqs. (10) and (11) denotes the time derivative. In the energy Eq. (11) $T_{i}$ and $n_{i}$ are the plasma temperature and density in the loop, $j_{i}$ is the density of the current, $\gamma=5 / 3$ is the adiabatic constant, $Q\left(T_{i}\right)$, the radiative loss function for optically thin emission (Cox \& Tucker 1969; Rosner et al. 1978; Peres et al. 1982), and $H_{i}$ is a stationary background heating introduced to provide thermal equilibrium in the initial steady state.

Applying again the thin torus approximation for the loops we have their inductances determined by Eq. (2), whereas for mutual inductance coefficients we use the approximate expression derived by Melrose (1997) and modified by Aschwanden et al. (1999a)

$M_{i j}=8\left(L_{i} L_{j}\right)^{1 / 2}\left[\frac{R_{\text {loop } i} R_{\text {loop } j}}{\left(R_{\text {loop } i}+R_{\text {loop } j}\right)^{2}+d_{i j}^{2}}\right] \cos \varphi_{i j}$,

where $L_{i}, L_{j}$ are the inductances of the loops (2), $R_{\text {loop }} i, R_{\text {loop j }}$, their major radii, $d_{i j}$, the distance between the centers of the loops' tori, and $\varphi_{i j}$, the angle between the normal vectors to the loops' planes. Here we neglect the curvature of the solar surface and suppose a vertical orientation of the loops, i.e., the angle between the plane of each loop and the normal vector to the surface of the Sun is zero. The uniform current profile assumed in the loops (i.e. $j_{i}=\frac{I_{i}}{\pi r_{0 i}^{2}}$, where $r_{0 i}$ is the radius of cross-section of a loop) appears to be a good approximation for a coronal magnetic tube with the force-free field $B_{z}(r)=$ $2 B_{0 i} \frac{1}{\left(r / r_{0}\right)^{2}+1}, \quad B_{\varphi}(r)=2 B_{0 i} \frac{r / r_{0 i}}{\left(r / r_{0}\right)^{2}+1}$ at large distances, $r \gg r_{0 i}$. This fact is essential for the correct estimation of the mutual inductances of real loops in active regions (Khodachenko \& Zaitsev 1998).

To include the effects of inductive build up of the current in a rising magnetic loop into the model, we consider a situation where the initially current-free magnetic loop (No. 2) grows in the vicinity of a current-carrying loop (No. 1). For a more general view we assume that both loops rise, but the initially smaller current-free loop grows faster than the bigger one. We consider a linear increase in time of the major radii of the loops: $R_{\text {loop } i}=R_{\operatorname{loop} i}^{0}+v_{i} t, \quad i=1,2$. The assumption of the initial steady state and thermal equilibrium of the loops yields

$$
\begin{aligned}
& U_{01}=R_{1}(t=0) I_{01}, \quad U_{02}=R_{2}(t=0) I_{02}=0, \\
& H_{1}=n_{1}^{2} Q\left(T_{1}(t=0)\right)-\frac{I_{01}^{2}}{\left(\pi r_{01}^{2}\right)^{2} \sigma\left(T_{1}(t=0)\right)}, \\
& H_{2}=n_{2}^{2} Q\left(T_{2}(t=0)\right)-\frac{I_{02}^{2}}{\left(\pi r_{02}^{2}\right)^{2} \sigma\left(T_{2}(t=0)\right)}=n_{2}^{2} Q\left(T_{2}(t=0)\right),
\end{aligned}
$$

where $I_{01}$ and $I_{02}=0$ are the initial values of current in the loops. The initial resistances $R_{i}(t=0)$ of the loops are calculated from their initial geometry. In Figs. 3 and 4 the dynamics of the current and plasma temperature in the first (Figs. 3a and 4a) and second (Figs. 3b and 4b) loop are shown. These graphs represent the numerical solution of the set of Eqs. (10) and (11) written for each loop. By this, Fig. 3 presents the dependence of the currents and plasma temperature evolution on the value of the angle $\varphi_{12}$ between the loops with $R_{\text {loop } 1}^{0}=2 \times 10^{9} \mathrm{~cm}, R_{\text {loop } 2}^{0}=10^{8} \mathrm{~cm}, r_{01}=r_{02}=5 \times 10^{7} \mathrm{~cm}$, $v_{1}=5 \times 10^{5} \mathrm{~cm} \mathrm{~s}^{-1}, v_{2}=10 \times 10^{5} \mathrm{~cm} \mathrm{~s}^{-1}, I_{01}=3 \times 10^{11} \mathrm{~A}$, $I_{02}=0 \mathrm{~A}, n_{1}=n_{2}=10^{9} \mathrm{~cm}^{-3}, T_{1}(t=0)=T_{2}(t=0)=10^{6} \mathrm{~K}$ and $d_{12}=2 \times 10^{8} \mathrm{~cm}$. In Fig. 4 the dependence of the dynamics of currents and plasma temperature on the distance $d_{12}$ between the loops is shown for the case of $\varphi_{12}=0$. The parameters of the loops here are the same as above, but a smaller initial current in the main loop, $I_{01}=10^{11} \mathrm{~A}$, is assumed.

According to the solution obtained, a current is excited in the initially current-free loop. This current is directed opposite to the initial current in the neighboring loop. The situation modelled here corresponds to a relatively fast rise of a new magnetic flux near a preexisting larger, current-carrying magnetic structure. From Fig. 3 one can see that the parallel loops $\left(\varphi_{12}=0\right)$ interact most effectively. This is a reasonable result since inductive interaction only takes place between currents which are not orthogonal to each other. The interaction is enhanced by decreasing the distance between the interacting currents. This is well demonstrated in Fig. 4. In principle, the dynamics of currents and plasma temperature similar to the one shown in Fig. 4 can be also obtained in the case of a non-zero $\varphi_{12}$, but this will require higher initial currents in the loops, or/and higher rising speeds, or/and shorter distances $d_{12}$.

According to the above numerical solutions (see Figs. 3 and 4), the behaviour of currents in both interacting loops has an 

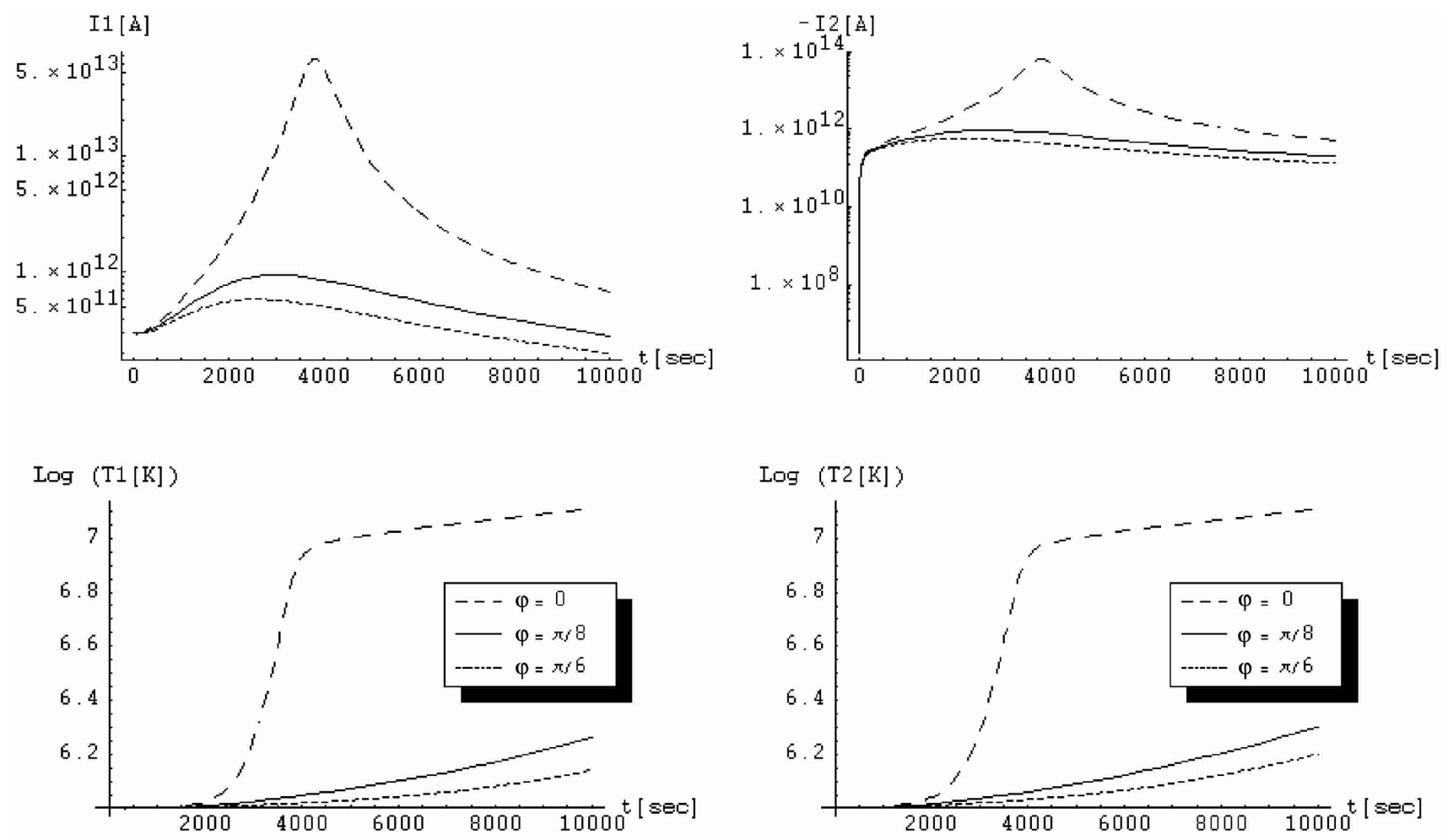

a

$\mathrm{b}$

Fig. 3. Dynamics of currents and plasma temperature in two inductively connected growing vertical loops for different angles $\varphi_{12}$.

impulsive character with a maximum value reached at the moment when the size of the faster growing loop becomes about the size of the initially bigger and slower loop. By this, peak values of the current can exceed $10^{14} \mathrm{~A}$. The maximum current correlates in time with the fast plasma temperature increase in the loops up to values of $(2-5) \times 10^{7} \mathrm{~K}$. This looks like a thermal flare taking place in the loops. Here however it is not necessary that the loops reconnect with each other. At the same time, if the loops are sufficiently close to each other, $d_{12} \leq r_{01}+r_{02}$ (in our calculations the case with $d_{12}=500 \mathrm{~km}$ ), they will reconnect when their heights become equal. In this case the scenario of a flare suggested by Melrose (1997) can be realized. But when reconnecting, the loops will have already very high currents, and the temperature of the loops will be much higher than the typical coronal value. This fact should be taken into account when applying the quadrupolar reconnection model of a flare.

One more interesting feature of the strong inductive interaction of relatively moving current-carrying loops consists of the high value of the vortical inductive electric field generated within the loops. This field in the $i$ th loop can be roughly estimated as $E=\frac{I_{i}^{2}}{\left(\pi r_{0 j}^{2}\right)^{2} \sigma\left(T_{i}\right)}$. In principle, this situation is possible when the value of the inductive electric field exceeds the critical value of the electric field $E_{\mathrm{c}} \approx 0.2 \frac{4 \pi n e^{3}}{k_{B} T} L_{\mathrm{e}}$ for which electrons are in a run-away regime. Here $L_{\mathrm{e}}$ is Coulomb logarithm. In Fig. 5 the dynamics of the electric fields $E$ and $E_{\mathrm{c}}$ are presented for the case of the growing parallel $\left(\varphi_{12}=0\right)$ loops considered above. Since the conditions (currents and plasma temperature) in both interacting loops become similar very quickly (see Fig. 4), we plot the electric fields only for the slow loop. It follows from Fig. 5 that the acceleration of the run-away electrons becomes possible in all cases after time $t \sim 2000 \mathrm{~s}$. The values of the inductive electric field $E$ are sufficient to accelerate the electrons on the loop's length, $l_{\text {loop }}$, up to the energies $W_{\mathrm{e}}[\mathrm{eV}]=E[\mathrm{~V} / \mathrm{m}] \cdot l_{\text {loop }}[\mathrm{m}]=100-1000 \mathrm{keV}$. Of course, this is a very rough estimation, but it shows that the generation of intensive beams of fast particles is quite possible during the inductive interaction of sufficiently close, currentcarrying, coronal magnetic loops which move relative to each other. Moreover, according to this model, preliminary beams of fast electrons can be generated in the system of two interacting loops some time before the loops intersect and the reconnection process starts. Note however that this effect requires sufficiently high values of currents in the loops $\sim 10^{10}-10^{11} \mathrm{~A}$, which are likely to occur in rapidly evolving solar active regions, but seem to be less typical for magnetic loops of the quiet Sun.

Analogous acceleration of run-away electrons can also take place in the case when a separatrix current changes its path during a preflare evolution of a 3D magnetic helmet structure. As was demonstrated above, the change of a magnetic flux through the current circuit sometimes generates large inductive electric fields which can accelerate particles and produce flaring beams.

\section{Dynamic interaction of inductively connected current-carrying magnetic loops}

\subsection{General approach to the problem}

In the previous section we considered the effects of the electric current and the temperature change in a system of 

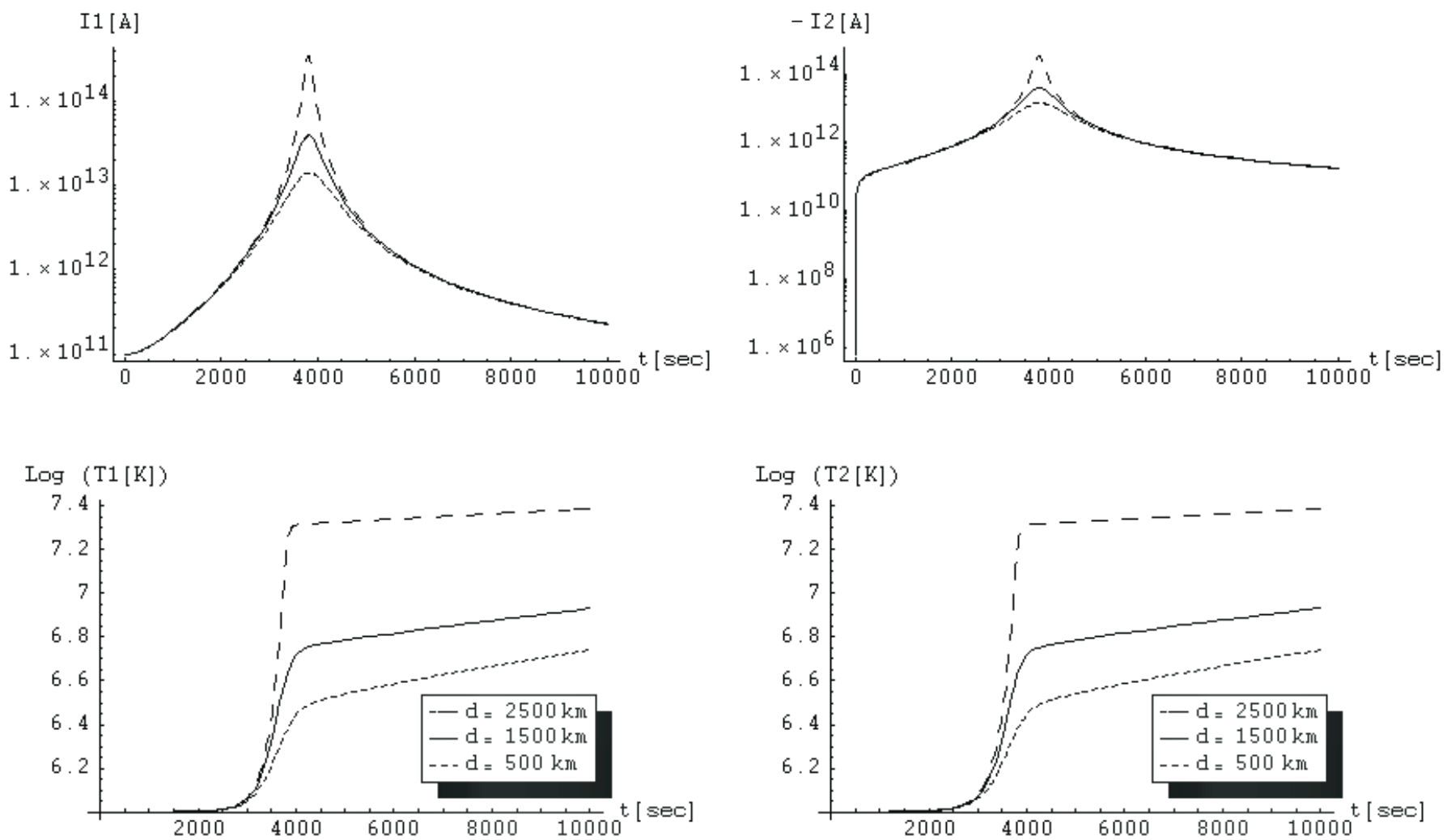

a

$\mathrm{b}$

Fig. 4. Dynamics of currents and plasma temperature in two inductively connected growing up vertical and parallel to each other loops in dependence on the distance $d_{12}$ between them.

inductively connected moving current-carrying loops. These effects are caused by the inductive electromotive forces occurring in the electric circuits of the loops and significantly influencing the current flow, which in turn has an effect on the energy balance. By this, the orientation of the loops with respect to the solar surface and each other was prescribed and assumed to be constant.

Now let us consider the effects of the dynamic coupling of magnetically connected current-carrying loops which probably influence the mutual orientation of the loops in reality. Besides, these effects can induce specific dynamics of coronal magnetic loops, such as oscillations or change of inclination.

As mentioned above, each pair of current-carrying magnetic loops is coupled dynamically by a $\frac{1}{c}[\boldsymbol{j} \times \boldsymbol{B}]$ force. Considering the loops as electric circuits with flowing currents, we can find a generalized ponderomotive force of their interaction

$F_{i}=-\frac{\partial U}{\partial x_{i}}$

where

$U=-\frac{1}{2 c^{2}} \sum_{i, j} M_{i j} I_{i} I_{j}$

is a potential force function of the system of currents, $M_{i j}=$ $M_{j i}, M_{i i} \equiv L_{i}$, inductive coefficients, and $x_{i}$, a generalized coordinate (for example the angle between the loops' planes). Note that when the motion of a dynamic system is described by the generalized coordinates in terms of generalized forces $F_{i}$ and momenta $P_{i}$ the equations of motion are still $\dot{P}_{i}=F_{i}$. Only in Cartesian coordinates the generalized momenta are the components of vectors $m v_{i}$ (usual mechanical momenta). In general, however, the $P_{i}$ does not reduce to products of mass and velocity and therefore the dimension of the generalized force $F_{i}$ could be different from the one of the usual mechanical force (Landau \& Lifshitz 1960b, p. 16). The potential force function $U$ should not be identified with a magnetic potential energy, $W_{\mathrm{M}}$, of a system of currents, since any motions of a conductor in a magnetic field are accompanied not only by the work of the magnetic ponderomotive forces, but also by the work of electromotive forces induced in a moving conductor. Therefore, the change in energy of the magnetic field during motion of a conductor cannot be determined from the work of only ponderomotive forces. From the theory of magnetism of currents it follows that for quasi-stationary currents $U=-W_{\mathrm{M}}$.

The whole energy balance in a system of inductively interacting quasi-stationary currents is described by the following equation

$\mathrm{d} W_{\mathrm{M}}+A=(P-Q) \mathrm{d} t$

where $A$ is the mechanical work produced during the motion of current-carrying conductors, $Q$ is the energy released due to the dissipation mechanisms in the system and $P$ is the work of the external electromotive forces of the current sources. 

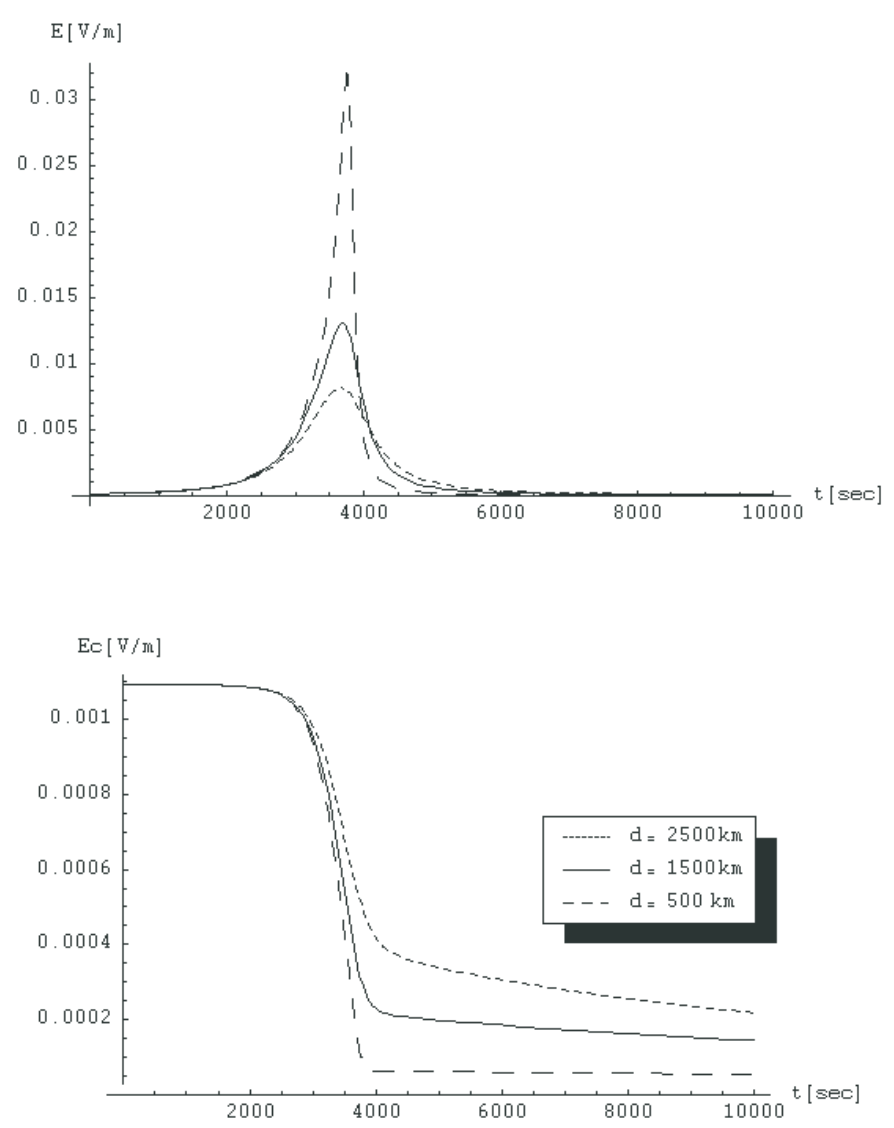

Fig. 5. Dynamics of the electric fields $E$ and $E_{\mathrm{c}}$ in the case of two growing up inductively coupled parallel $\left(\varphi_{12}=0\right)$ current-carrying magnetic loops.

Equation (15), in particular, states that in the case of moving conductors the energy of external sources is spent on the mechanical work, heating, and change of the magnetic energy of the current system.

The advantage of the potential force function is that it simplifies significantly the analysis of the ponderomotoric interaction of currents. It allows one to avoid the complex integration of forces, acting on each separate element of a current. In particular, as follows from Eq. (13), a stable equilibrium of a system of current circuits corresponds to a minimum of its potential force function $U$. Therefore, the traditional formalism of mechanics, developed for dynamic systems in potential fields can easy be applied to the analysis of dynamic interaction of the inductively connected coronal current-carrying loops.

Below we consider a system consisting of one main loop in the center and two minor lateral loops located at a distance $d$ on both sides of the main loop (see Fig. 6). The centers of the loops are located on the same axis, but their planes are inclined at angles $\Theta_{i}$ with the vertical direction. The lateral loops are assumed to be symmetrically inclined at the same angle, but in different directions, i.e. $\Theta_{1}=-\Theta_{3}$. We approximate each loop by a rectangular structure characterized by height $h_{i}$, length $b_{i}$, and thickness $2 r_{0 i}$. The longitudinal currents in the loops are again assumed to be homogeneously distributed over

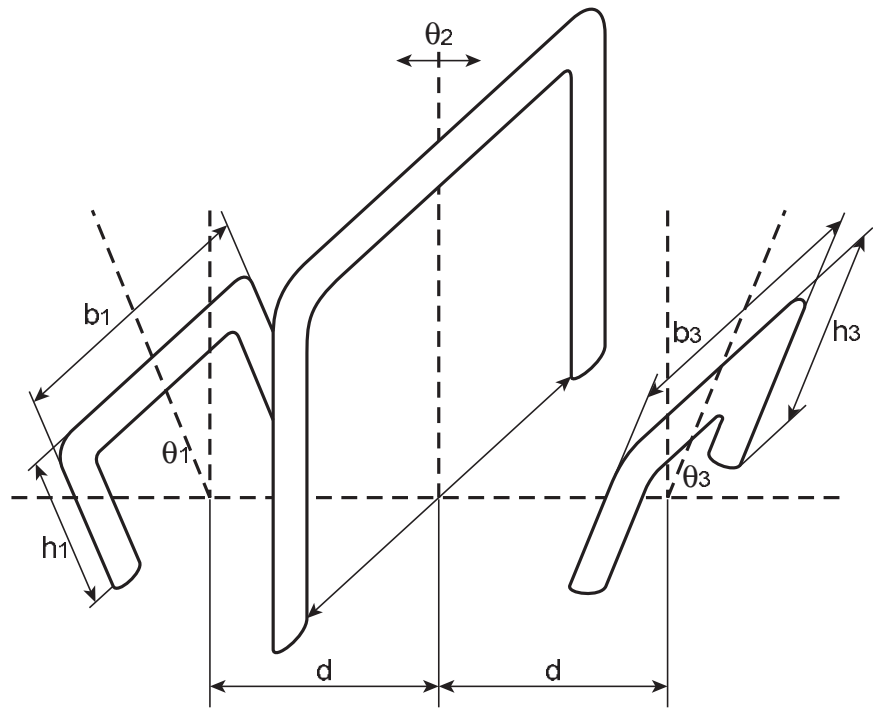

Fig. 6. Schematic picture of the system consisting of one main loop in the center and two minor lateral loops.

a cross-section of a magnetic tube. The simplified geometry of the loops allows one to calculate from the general formulae,

$L_{i}=\frac{1}{I_{i}^{2}} \int_{V_{i}} \int_{V_{i}} \frac{j_{i} \boldsymbol{j}_{i}^{\prime} \mathrm{d} V \mathrm{~d} V^{\prime}}{\left|\boldsymbol{R}_{i}\right|} \quad M_{i j}=\frac{1}{I_{i} I_{j}} \int_{V_{i}} \int_{V_{j}} \frac{\boldsymbol{j}_{i} \boldsymbol{j}_{j} \mathrm{~d} V_{i} \mathrm{~d} V_{j}}{\left|\boldsymbol{R}_{i j}\right|}$,

the precise expressions for inductance and mutual inductance coefficients, which are necessary for the dynamic analysis of the system. The approximate expression (12) is not accurate enough for this purpose.

To study the dynamic regimes of the system consisting of three interacting current-carrying loops it is sufficient to consider only a part of the whole potential force function $U$, the so called mutual potential force function

$U_{123}=-\frac{1}{c^{2}}\left(M_{12} I_{1} I_{2}+M_{23} I_{2} I_{3}+M_{13} I_{1} I_{3}\right)$.

The function $U_{123}$ describes the dynamic interaction of the loops and depends on their mutual orientation, whereas the rest of $U$,

$\tilde{U}_{123}=U-U_{123}=-\frac{1}{2 c^{2}}\left(L_{1} I_{1}^{2}+L_{2} I_{2}^{2}+L_{3} I_{3}^{2}\right)$,

appears just as a constant under the derivative (13).

In Fig. 7 the mutual potential force function of the system of three loops is shown as a function of the angles $\Theta_{2}=\Theta$ and $\Theta_{1}=-\Theta_{3}=\alpha$ for different relations between the size parameters of the central, $b_{2}=h_{2} \equiv s_{\mathrm{c}}$, and lateral, $b_{1}=$ $h_{1}=b_{3}=h_{3} \equiv s_{1}$, loops. In particular, Figs. 7a-7d correspond to $\frac{s_{\mathrm{c}}}{s_{1}}=50,10,5$, and $5 / 3$, respectively. Other parameters of the system are $s_{\mathrm{c}}=5 \times 10^{9} \mathrm{~cm}, d=5 \times 10^{8} \mathrm{~cm}, r_{0 i}=$ $5 \times 10^{7} \mathrm{~cm}, T_{i}=10^{6} \mathrm{~K}, i=1,2,3, I_{1}=I_{3}=-0.5 \times 10^{10} \mathrm{~A}$, and $I_{2}=10^{10} \AA$. The negative current in lateral loops (No. 1, No. 3) means that it is in the opposite direction to the current in the central loop (No. 2). As one can see, the function $U_{123}(\Theta, \alpha)$ always has a maximum at a point $\left(\Theta=0 ; \alpha=\alpha_{\text {eq }}\right)$, which corresponds to an unstable equilibrium. The variation of the value 

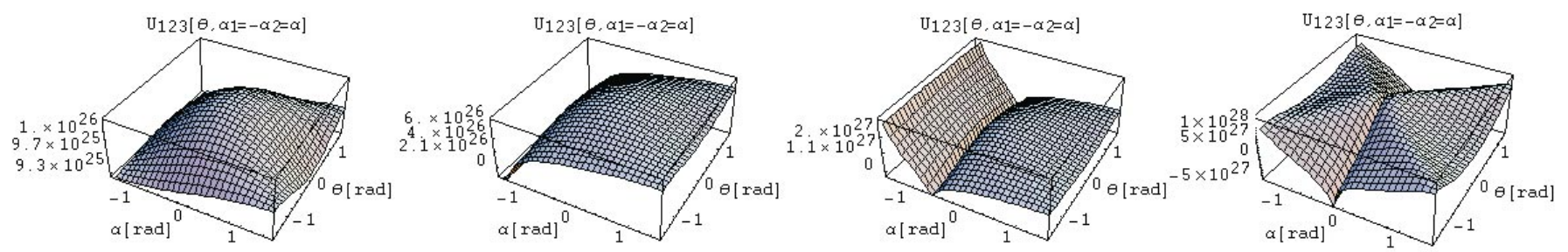

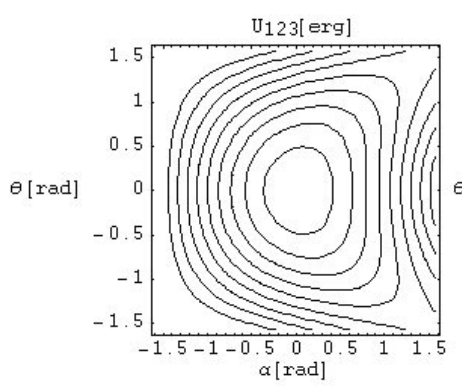

a

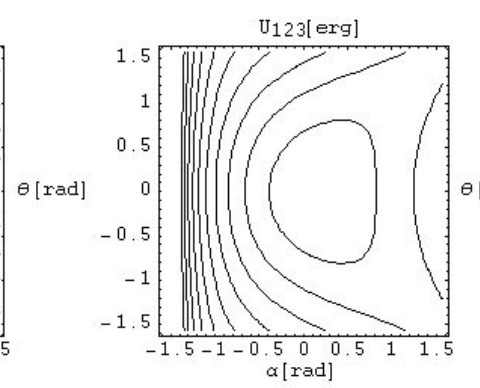

$\mathrm{b}$

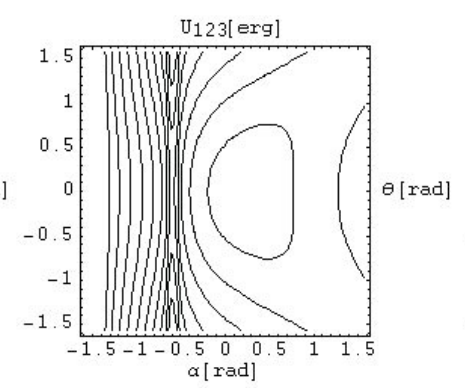

C

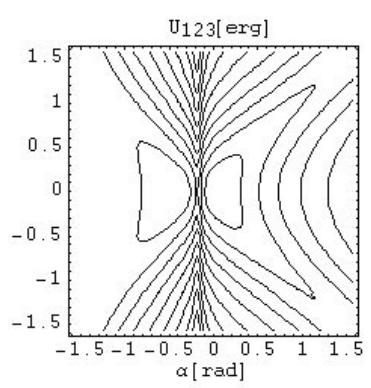

a

Fig. 7. Mutual potential force function $U_{123}$ of the system of three loops in dependence on the angles $\Theta$ and $\alpha$ for different relations between the size parameters of the central and lateral loops.

of $\alpha_{\text {eq }}$ with $s_{1}$ is presented in Fig. 8. The fact that this equilibrium is unstable means that the external disturbances produced by dynamic and flaring events in neighboring magnetic structures and propagating in the solar atmosphere can cause a relatively fast reconfiguration of the system resulting in a change of the inclination of the loops. By this, the final stationary values of the inclination angles of the loops will also depend upon the magnetic elasticity forces in the loops' footpoints where the magnetic field lines are fixed. In our analysis this complicated case is not considered. Instead, in the final steady state picture we take for the central loop $\Theta=0$ and assume some reasonable (in agreement with observations) value of $\alpha$ for the lateral loops.

Of course, the dynamic system considered here, consisting of only three current-carrying loops, appears to be a much simplified model of real magnetic structures on the Sun. Usually tens of magnetic loops are located close enough to each other to interact inductively. In the multiple loop system, conditions and features of the equilibrium configuration will certainly differ from those in our simple case. On the other hand, the dynamic effects which we study using the simple model should be basically the same.

\subsection{Dynamics of the central loop in the system of three current-carrying loops with growing lateral loops}

It follows from Fig. 7 that in the case of sufficiently large lateral loops and for a reasonably large fixed value of their inclination $\alpha$, the angle $\Theta=0$ corresponds to a stable equilibrium. To consider the formation and evolution of this equilibrium in the system of three inductively interacting magnetic loops (Fig. 6), we suppose that the initially current-free lateral magnetic loops rise near the central current-carrying loop. We assume a linear law for the increase of the lateral

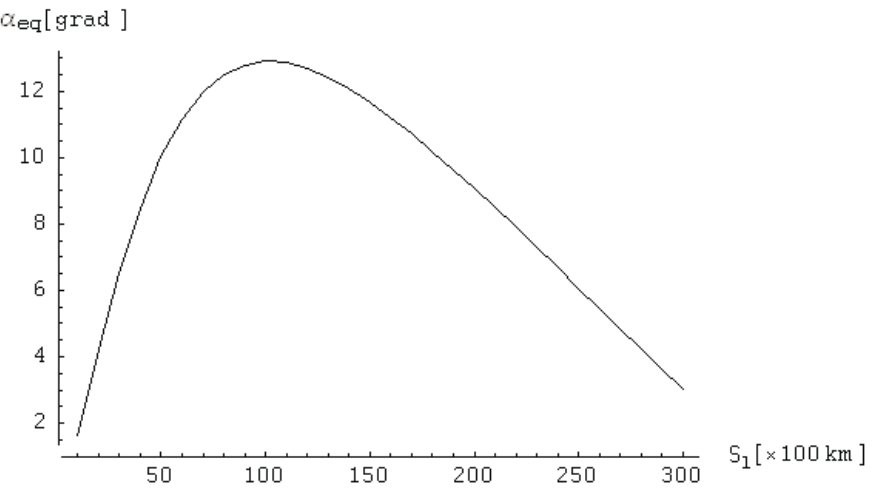

Fig. 8. Dependence of the value of $\alpha_{\mathrm{eq}}$ on $s_{1}$, characterizing the scale of the lateral loops.

loops, $h_{i}(t)=h_{0 i}+a_{i} t, \quad b_{i}(t)=b_{0 i}+a_{i} t$, where $i=1,3$ is the loop number. The evolution of currents in the loops is described by the set of three equations

$I_{i} R_{i}=U_{0 i}-\frac{1}{c^{2}} \sum_{j=1}^{3}\left(M_{i j} \dot{I}_{k}+I_{k} \dot{M}_{i j}\right)$

where $i$ takes the values from 1 to 3 and $M_{i i} \equiv L_{i}$. From the initial state with zero currents in the lateral loops and a given current in the central loop we obtain $U_{0 i}=R_{i}(t=0) I_{0 i}=0, i=$ 1,3 and $U_{02}=R_{2}(t=0) I_{02}$. The Eqs. (19) are solved numerically for the following parameters of the model: $\Theta_{1}=-\Theta_{3}=$ $\pi / 4, \Theta_{2}=0, h_{01}=h_{03}=5 \times 10^{8} \mathrm{~cm}, b_{01}=b_{03}=5 \times 10^{8} \mathrm{~cm}$, $h_{02}=b_{02}=3 \times 10^{9} \mathrm{~cm}, d=5 \times 10^{8} \mathrm{~cm}, r_{0 i}=5 \times 10^{7} \mathrm{~cm}, T_{i}=$ $10^{6} \mathrm{~K}, i=1,2,3, a_{1}=a_{3}=1.5 \times 10^{5} \mathrm{~cm} \mathrm{~s}^{-1}$, and $I_{02}=10^{4} \AA$. Large longitudinal currents are typical for magnetic loops in the flaring solar active regions, whereas in this section we model a group of loops in the quiet Sun. So, a relatively small value for the initial current in the central loop is assumed. At the same time, as follows from the linearity of the set of Eqs. (19) with 
respect to the current, the solutions of Eqs. (19) will have a similar structure for any initial current $I_{02}$ and corresponding external electromotive force $U_{02}$. The difference will only be in the absolute numerical values obtained in each case, which are proportional to $I_{02}$. According to the numerical solution of the set of Eqs. (19), the dynamics of currents in the loops look similar to those obtained in Sect. 3 for the case of two interacting current-carrying loops. All the currents again reach maximum values when the size of the rising lateral loops becomes about the size of the central loop. In Fig. 9 the temporal evolution of the mutual potential force function $U_{123}$, depending also on the angle of inclination of the central loop $\Theta_{2}=\Theta$, is shown. It again confirms the conclusion following from Fig. 7, that for given fixed inclinations of the lateral loops the vertical central loop is in a stable equilibrium. Judging by the shape of the function $U_{123}(\Theta, t)$, two types of motion of the central loop are possible, depending on the amount of the energy supplied to the system from outside. These could be oscillations of the loop near the equilibrium vertical position, or fast increases of its inclination. The latter happens in the case when an external disturbance makes the system overcome the local maximum of $U_{123}(\Theta, t)$, corresponding to $\Theta_{\max } \approx \pm 0.9 \mathrm{rad}$. Sufficiently strong neighboring flaring events and shocks propagating in the coronal plasma can appear as a possible source, providing the system's external energy input.

\subsection{Oscillations of a current-carrying loop}

Here we concentrate on the oscillatory regime of the central loop in the system of three inductively interacting loops considered in the previous subsection. Excitation of oscillations of the central loop requires less disturbing energy input than the process of fast increase of inclination of the loop. Therefore, oscillations are more easily caused. Moreover, recent TRACE observations at extreme-ultraviolet wavelengths (171 ̊) detected oscillations of the loops which usually happened simultaneously with a neighboring flaring event (Aschwanden et al. 1999b; De Moortel et al. 2000). Oscillations of the loops are traditionally explained as standing, or propagating MHD waves. Among the most probable candidates are a standing fast kink mode (Aschwanden et al. 1999b), or a slow magnetoacoustic wave (De Moortel et al. 2000). Oscillations of the loops which are possible in our model are not MHD in nature. They are caused by the ponderomotoric interaction of currents in groups of inductively coupled current-carrying loops. Here we show that under the favourable parameters of the model (value of the currents; geometric scales of the loops; energy input into the system), all basic observed characteristics of oscillations of the coronal loops can be reproduced well. Thus, the ponderomotoric interaction of current-carrying loops can be considered as one more possible way to explain the observed phenomenon of oscillations of solar magnetic loops.

To estimate the main characteristics of the oscillatory regime of the central loop in our model, we assume that the oscillation periods $P_{\text {osc }}$ are not very long and/or amplitudes are small enough. Thus, we can neglect the influence of a changing orientation of the oscillating central loop on the global currents
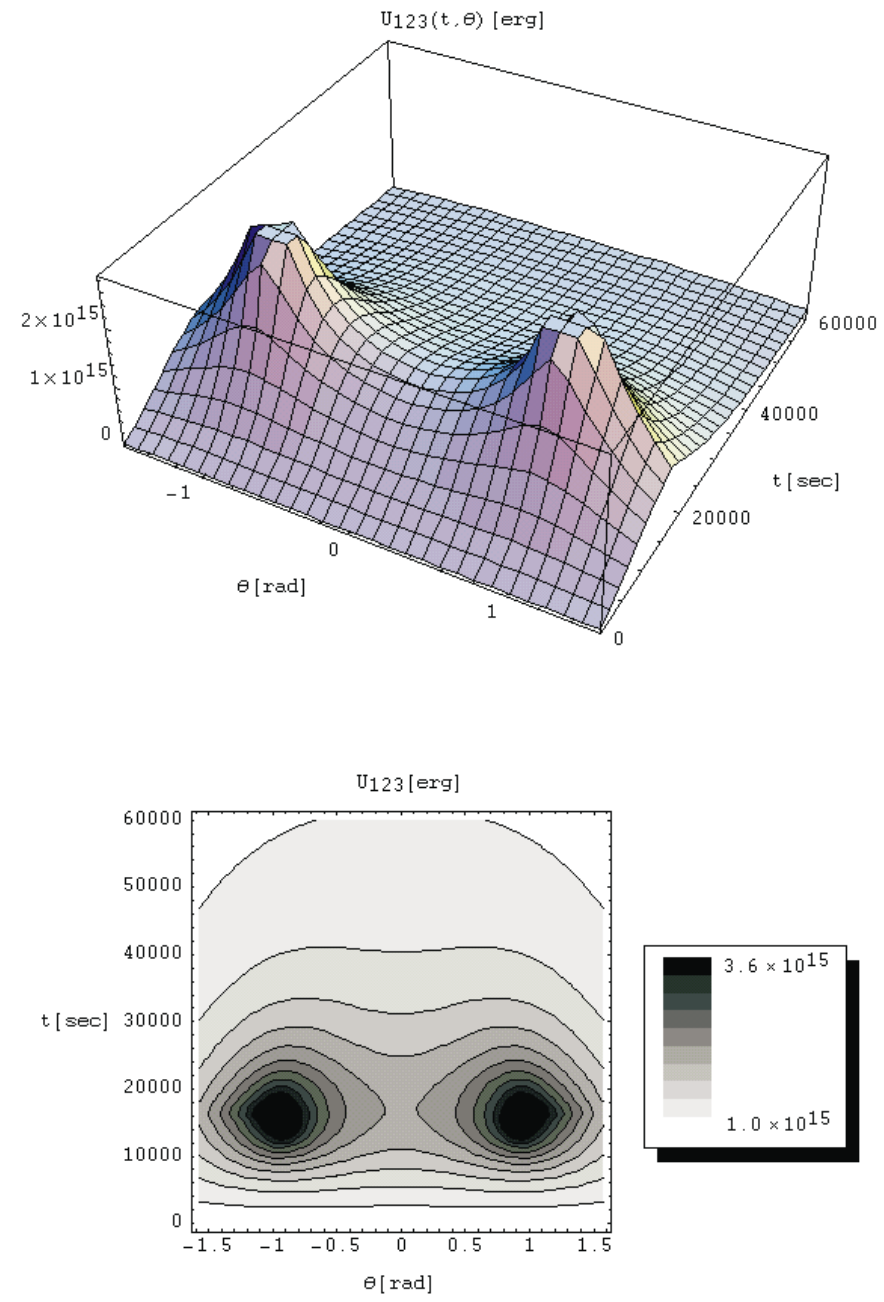

Fig. 9. Temporal evolution of the mutual potential force function $U_{123}$, depending as well on the angle of inclination of the central loop $\Theta_{2}=\Theta$.

dynamics in the system and use the quasistationary current approximation in our analysis. I.e. we consider the case when $P_{\text {osc }}<\tau_{\text {curr }}$, where $\tau_{\text {curr }}$ is the characteristic time of the current variation in the system. On the other hand, in order apply the electric circuit model for the current-carrying loops, $P_{\text {osc }}$ should be larger than the Alfvén propagation time $\tau_{\mathrm{A}} \sim 1-10 \mathrm{~s}$.

The period of oscillations of the system with a dipped potential profile $U_{123}\left(\Theta, t^{*}\right)$, can be calculated by the formula

$P_{\mathrm{osc}}=\sqrt{2 \mathcal{J}_{0}} \int_{\theta_{1}}^{\theta_{2}} \frac{\mathrm{d} \Theta}{\left(E_{\mathrm{ext}}-U_{123}\left(\Theta, t^{*}\right)\right)^{1 / 2}}$,

where $\mathcal{J}_{0}$ is the inertia momentum of the loop with fixed footpoints and $E_{\text {ext }}$ an external energy input, provided to the system by a disturbance (shock) propagating in the corona. To be specific we take here a particular form of the mutual potential force function $U_{123}\left(\Theta, t^{*}\right)$ (see Eq. (17) and Fig. 9) realized at the moment $t=t^{*} \approx 16000 \mathrm{~s}$, when the size of the growing lateral loops becomes equal to the size of the central loop. The reflecting points $\theta_{1}$ and $\theta_{2}$ correspond to $E_{\text {ext }}=U_{123}\left(\Theta, t^{*}\right)$. The amplitude $\Delta$ and velocity $v$ of the top point of the oscillating loop are determined by

$\Delta=h_{2} \sin \theta_{2}, \quad v=2 h_{2} \frac{\theta_{2}-\theta_{1}}{P_{\mathrm{osc}}}$. 
Table 1. Amplitude $(\delta \Theta$ and $\Delta)$, velocity $(v)$, and period of oscillations $\left(P_{\text {osc }}\right)$ of the coronal loop, calculated for different values of the disturbing energy $\left(E_{\text {ext }}\right)$.

\begin{tabular}{cccccccc}
\hline \hline$E_{\text {ext }}[\mathrm{erg}]$ & $1.29 \times 10^{15}$ & $1.38 \times 10^{15}$ & $1.46 \times 10^{15}$ & $1.55 \times 10^{15}$ & $1.63 \times 10^{15}$ & $1.72 \times 10^{15}$ & $1.80 \times 10^{15}$ \\
\hline$\delta \Theta$ & $1.03^{\circ}$ & $2.72^{\circ}$ & $4.40^{\circ}$ & $6.09^{\circ}$ & $7.78^{\circ}$ & $9.46^{\circ}$ & $11.1^{\circ}$ \\
$\Delta[\mathrm{km}]$ & 541 & 1420 & 2300 & 3180 & 4050 & 4930 & 5800 \\
$P_{\mathrm{osc}}[\mathrm{s}]$ & 119 & 194 & 247 & 292 & 331 & 366 & 398 \\
$v\left[\mathrm{~km} \mathrm{~s}^{-1}\right]$ & 18.1 & 29.3 & 37.2 & 43.6 & 49.2 & 54.1 & 58.6 \\
\hline
\end{tabular}

Table 1 presents the main characteristics of the oscillatory regime of the loop, calculated by Eqs. (20) and (21) for different values of a disturbing energy input. The results obtained are rather close to the observed parameters of the coronal magnetic loops oscillations (Aschwanden et al. 1999b). As for the MHD waves propagating along the loop, this also can take place in our case, but as a kind of a secondary effect, arising during the loop motion under the action of the ponderomotive forces. It is natural to expect that the whole large loop will move not as a rigid object, but having local transverse deformations, which can appear as the origin of MHD waves travelling along the loop. Looking at the numerical value of the obtained possible periods of the loop oscillations, we note that these numbers are rather flexible and according to Eqs. (17) and (20) depend on the values of currents in the loops, defined by the initial current $I_{02}$. Because of the linearity of the Eqs. (19), for each new value of $I_{02}$, the period of the oscillations changes as $\propto I_{02}^{-1}$, whereas the corresponding disturbing energy input should be taken $\propto I_{02}^{2}$. This means, in particular, that the loops with lower currents can more easily be disturbed, since they require a lower energy of the disturbing signal. By this, the velocity of the top of the loop changes as $\propto I_{02}$.

The observed decay of oscillations of solar magnetic loops which is usually explained by damping of a corresponding MHD mode (Nakariakov et al. 1999), or by phase effects in groups of oscillating loops (Schrijver \& Brown 2000), can also be interpreted within the framework of the model taking into account the ponderomotoric interaction of the loops. For example, oscillations of the central loop in our case will gradually vanish if this loop is disturbed at the moment when the whole system just evolves to the state with a maximum potential dip, realized at $t \approx 16000 \mathrm{~s}$. Because of the temporal increase of the value $U_{123}(\Theta=0, t)$ in the potential dip for $t \leq 16000 \mathrm{~s}$ (see Fig. 9), the given initial disturbing energy after some time can become insufficient to support the oscillations and they will vanish.

\section{Summary}

In conclusion we summarize the basic idea of this paper and the main results.

- In spite of the observational data of the vector magnetic field indicating the presence of unneutralized currents in coronal magnetic loops, there appears to be no theory explaining how such currents can be generated after the loops emergence from below the photosphere. Here we consider one of the possible mechanisms which could influence, and even cause, the currents flowing in the coronal magnetic loops. This mechanism is based upon the effects of the inductive electromagnetic interaction between relatively moving (rising and growing) neighboring magnetic loops and employs the circuit model of a current-carrying magnetic loop. The relative motion of inductively coupled current-carrying magnetic loops causes the appearance of strong inductive electromotive forces in their electric circuits. These electromotive forces influence significantly the currents in the loops.

- The parameters of the solar coronal plasma and the typical geometric characteristics of magnetic loops cause a very large characteristic time scale $\tau=\frac{L}{R c^{2}}$ for the current change in an electric circuit of a loop. However, we have shown here that in the case of a rising and growing magnetic loop the dynamics of the current in the circuit is completely defined by the dynamics of the inductance of the loop (i.e., the change in size and shape of the loop).

- To make the multiple-loop equivalent circuit approach more realistic, we take into account, in some of our models, that temporal changes of the lengths of inductively coupled current-carrying loops cause changes of currents in the loops and disturb their initial thermal equilibrium. The change of the plasma temperature in current channels of the loops influences in its turn the plasma conductivity and therefore, the resistances in the equivalent electric circuits.

- In the example with two inductively connected loops, moving relative to each other, the dynamics of currents in both loops have an impulsive character with a maximum value $\sim 10^{14} \AA$ reached at the moment when the loops become equal in size. The maximum current correlates in time with a fast plasma temperature increase in the loops up to values $(2-5) \times 10^{7} \mathrm{~K}$, which looks like a thermal flare. For such a flare it is not necessary that reconnection of the loops takes place.

- Due to the inductive interaction of rising current-carrying loops with sufficiently high currents $\sim 10^{10}-10^{11} \AA$ (loops in an active region), acceleration of a large number of runaway electrons by the inductive electric field, up to energies of $\sim 100-1000 \mathrm{keV}$ is possible. Analogous acceleration of run-away electrons can also take place in the case when a separatrix current changes its shape during the preflare evolution of a 3D magnetic helmet structure.

- Ponderomotoric interaction of current-carrying magnetic loops causes dynamic coupling of the loops. It provides the possibility of oscillations of the loops, or the conditions for a fast change of their inclination (with possible reconnection following). Realization of each regime depends upon the amount of the external energy disturbing the system. 
A possible source of this external energy input can be flaring and explosive events, which generate shocks propagating in the coronal plasma.

- Oscillations of magnetic loops considered in our model have no relation to MHD waves, traditionally applied as the explanation of this type of phenomena. They are caused by the ponderomotoric interaction of currents in groups of inductively coupled current-carrying loops. By this, for certain parameters of the model (values of currents; geometric scales of the loops; disturbing energy input), all the basic observed characteristics of the loops oscillations can be well reproduced.

It should be clear however, that the models considered above are still very idealized approximations of real magnetic loops and their dynamics on the Sun. First of all, the number of interacting loops in a real situation is certainly much higher than it was in our models. At the same time, there always exists the possibility to associate bunches of real magnetic loops, located sufficiently close to each other and moving together, with one equivalent modelling loop, and therefore to split the complex magnetic structure into a smaller amount of interacting components. On the other hand, consideration of inductive interaction of multiple current-carrying loops in solar active regions opens a wide perspective for both theoretical physics and numerical techniques. Some steps in that direction were made by Khodachenko \& Zaitsev (1998), where the energetic processes in a random ensemble of inductively interacting currentcarrying loops were considered.

Acknowledgements. M. L. Khodachenko is grateful to the MaxPlanck-Gesellschaft and Austrian Academy of Sciences (ÖLZELT grant) for supporting his work. The authors express their special thanks to F. C. Cooper and D. Langmayr for assistance in the editing of the manuscript text.

\section{References}

Alfvén, H., \& Carlqvist, P. 1967, Sol. Phys., 1, 220

Aschwanden, M. J., Kosugi, T., Hanaoka, Y., Nishio, M., \& Melrose, D. B. 1999a, ApJ, 526, 1026

Aschwanden, M. J., Fletcher, L., Schrijver, C. J., \& Alexander, D. 1999b, ApJ, 520, 880
Canfield, R. C., de La Beaujardiere, J.-F., Fan, Y., et al. 1993, ApJ, 411,362

Canfield, R. C., Fan, Y., Leka, K. D., et al. 1990, preprint

Cox, D. P., \& Tucker, W. H. 1969, ApJ, 157, 1157

Ding, Y. J., Hagyard, M. J., DeLoach, A. C., Hong, Q. F., \& Liu, X. P. 1987, Sol. Phys., 109, 307

Gary, G. A., \& Demoulin, P. 1995, ApJ, 445, 982

Hagyard, M. J. 1989, Sol. Phys., 115, 107

Hanaoka, Y. 1996, Sol. Phys., 165, 275

Hanaoka, Y. 1997, Sol. Phys., 173, 319

Handy, B. N., Acton, L. W., Kankelborg, C. C., et al. 1999, Sol. Phys., 187, 229

Hardy, S. J., Melrose, D. B., \& Hudson, H. S. 1998, Publ. Astron. Soc. Aust., 15, 318

Jones, C. A., \& Galloway, D. J. 1993, J. Fluid Mech., 253, 297

Khodachenko, M. L. 1996, Astron. Rep., 40, No. 2, 252

Khodachenko, M. L., \& Zaitsev, V. V. 1998, Astron. Rep., 42, No. 2, 265

Landau, L. D., \& Lifshitz, E. M. 1960, Electrodynamics of Continuous Media (Oxford: Pergamon)

Landau, L. D., \& Lifshitz, E. M. 1960, Mechanics (Oxford: Pergamon)

Leka, K. D., Canfield, R. C., McClymont, A. N., \& van Driel-Gesztelyi, L. 1996, ApJ, 462, 547

Melrose, D. B. 1991, ApJ, 381, 306

Melrose, D. B. 1992, ApJ, 387, 402

Melrose, D. B. 1995, ApJ, 451, 391

Melrose, D. B. 1996, ApJ, 471, 497

Melrose, D. B. 1997, ApJ, 486, 521

De Moortel, I., Ireland, J., \& Walsh, R. W. 2000, A\&A, 355, L23

Moreton, G. E., \& Severny, A. B. 1968, Sol. Phys., 3, 282

Nakariakov, V. M., Ofman, L., DeLuca, E. E, Roberts, B., \& Davila, J. M. 1999, Science, 285, 862

Nishio, M., Yaji, K., Kosugi, T., Nakajima, H., \& Sakurai, T. 1997, ApJ, 489, 976

Parker, E. N. 1996, ApJ, 471, 489

Peres, G., Rosner, R., Serio, S., \& Vaiana, G. S. 1982, ApJ, 252, 791

Rosner, R., Tucker, W. H., \& Vaiana, G. S. 1978, ApJ, 220, 643

Schrijver, C. J., \& Brown, D. S. 2000, ApJ, 537, L69

Spicer, D. S. 1982, Space Sci. Rev., 31, 351

Wang, H., Ewell, M. W., Zirin, H., \& Ai, G. 1994, ApJ, 424, 436

Wang, J., Shi, Z., Wang, H., \& Lü, Y. 1996, ApJ, 456, 861

Zaitsev, V. V., \& Khodachenko, M. L. 1997, Radiophys. \& Quantum Electronics, 40, Nos. 1-2, 114

Zaitsev, V. V., Parfenov, O. G., \& Stepanov, A. V. 1978, Sol. Phys., 60, 279 